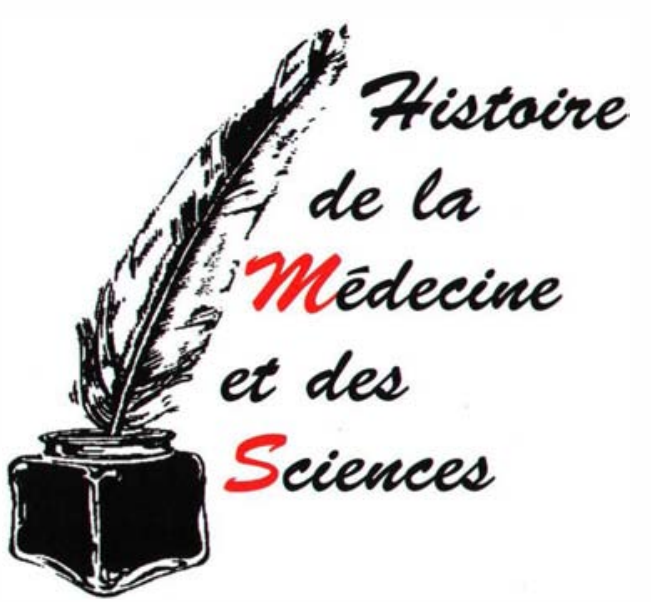

\section{De la danse de Saint Guy à la chorée de Huntington : rappels sur l'émergence d'un concept médical}

$\mathrm{L}$ es maladies caractérisées par des mouvements involontaires irrépressibles, et parmi elles la maladie (ou chorée) de Huntington font aujourd'hui partie des syndromes neurologiques bien définis sur le plan sémiologique. Cette identification est toutefois un fait récent, ce dont on pourrait s'étonner au vu des symptômes évidents présentés par les patients. L'histoire de la maladie de Huntington permet de mieux comprendre comment une affection de ce type a pu rester cachée durant de nombreux siècles, associée à des atteintes fort différentes ou considérée dans un cadre religieux plutôt que médical. La reconstitution de cette histoire a fait émerger des histoires personnelles qui, confrontées aux cadres socio-culturels et scientifiques des différentes époques, sont autant de démonstrations de l'image qu'une maladie se voit imposer par une époque.

Si les Égyptiens ont pensé dès 3000 av. J.-C. qu'un trouble de la motricité pouvait être dû à un dysfonctionnement cérébral, ils n'ont fait aucun commentaire à propos des mouvements involontaires. De même, ni Hippocrate ni Gallien n'ont mentionné ce type de problème. L'histoire de ces désordres marqués par l'apparition d'anomalies expressives de la motricité a débuté au MoyenAge en Europe occidentale continentale. C'est au XV $\mathrm{XV}^{\mathrm{e}}$ siècle, en Europe, que le terme chorée fut employé pour la première fois dans un sens affection caractérisée par une agitation involontaire, irrépressible, d'allure dansante: "la chorée de saint Vitus ou chorée de saint Jean". La plus ancienne citation connue de ce vocable provient d'une ordonnance de 1485 de la cité de Rottweil où il y est dit que: "quiconque appelle sur un autre la malédiction de la chorée de saint Vitus devra payer une amende de 6 shillings" [1]. Jusqu'à aujourd'hui, "avoir la danse de saint Guy " désigne, en langage populaire, l'existence d'une agitation choréiforme.

\section{Étymologie}

Le mot chorée provient du latin chorus et du grec choros et désignait chez ces derniers les danses sacrées que les disciples du culte d'Orphée pratiquaient autour des malades. Quant à son association avec saint Vitus et saint Jean, la tradition hagiographique apporte quelques hypothèses. Saint Vitus, ou saint Guy, serait né en 303 à Lucania dans le sud de l'Italie. Selon une légende, avant de mourir, il aurait supplié Dieu de préserver de toute affection choréique ou convulsante ceux qui célébreraient l'anniversaire de sa mort. Vénéré en Italie, Charlemagne encouragea son culte dans tout son Empire pour lutter contre les pratiques païennes; Charles IV le fit Saint Patron de Bohème et lui consacra la cathédrale de Prague; et, peu après, saint Vitus fut rangé parmi les 14 saints que les gens imploraient lorsqu'ils étaient malades. Quant à saint Jean (le Baptiste), son culte fut associé à un rituel de purification par le feu, peut-être inspiré de la Bible (voir les Evangiles de St Mathieu 3: 11, 12 et St Marc 1: 7,8 ) ou peut-être en souvenir de la danse que Salomé exécuta et qui lui coûta la vie. Sa fête, célébrée le 24 juin, fut intriquée avec d'anciennes traditions germaniques, comme la nodfyr, où les gens sautaient par-dessus le feu et la fumée pour être protégés une année entière des fièvres et autres maladies [2].

\section{Les manies dansantes}

L'histoire médicale de la chorée a commencé au début du deuxième millénaire, bien avant que la dénomination n'apparaisse, par un étrange fléau en Rhénanie germanique [3, 4]. Il était caractérisé par un besoin impérieux et irrépressible de danser, une grande suggestibilité du phénomène et une tendance à se propager sur un mode épidémique.

En 1021 à Kölbigk, près de Bernburg (Anhalt), après quatre ans de malheurs successifs, dix-huit hommes et femmes se mirent à danser et à chanter dans un pré près de l'église la nuit de Noël. Ils continuèrent sans pouvoir s'arrêter. Certains décédèrent de fatigue, d'autres furent atteints de tremblement jusqu'à la fin de leur vie. Plusieurs épisodes du même type eurent lieu au cours des siècles suivants. Mais, le plus marquant débuta à Aix-la-Chapelle en juillet 1374. Il nous est bien connu 
grâce au témoignage que le père Herenthal nous a laissé (cité par Hecker [3]). "Les danseurs formèrent des processions de plus en plus importantes, allant d'une ville à l'autre: Cologne, Frankfurt, Zurich. Ils se propagèrent en direction de l'ouest vers la Moselle et la Wallonie: Luxembourg, Metz (1 100 danseurs), Maastricht, Tongeren... et jusqu'à Liège qui fut atteinte en septembre. Partout ils trouvèrent des spectateurs qui, saisis par l'imitation, se joignirent à eux et les suivirent ". Pour la plupart des gens, les danseurs étaient des possédés. Leurs mouvements involontaires, leur état psychique particulier (vertiges, ivresses, hallucinations), leurs cris qui invoquaient des démons et leur projet d'égorger les prêtres qu'ils accusaient de fornication en étaient autant de démonstrations. Le remède appliqué fut la pratique d'exorcismes, de processions et la lecture de passages de l'Évangile. En quelques mois, l'épidémie fut maîtrisée. Cependant, des recrudescences estivales se produisirent. Elle reprit à Strasbourg en 1414 et se répandit dans le Bade-Wurtemberg et en Bavière. Cette fois le clergé fit appel à saint Vitus, qui devint, ensuite, celui auquel on s'adressait pour arrêter les danseurs, comme à Metz en 1463, Strasbourg encore en 1518, etc...

Le souvenir de ces manies dansantes peut être retrouvé dans certaines traditions populaires. Ainsi, la procession qui a lieu chaque année à la Pentecôte en la ville d'Echternach située entre Luxembourg et $\mathrm{St}$ Vith..., et qui est très semblable à celle représentée dans un dessin de Brueghel l'Ancien témoignant de l'épisode survenu en 1564 près de Bruxelles: "Les pèlerins de Muelebeeck" [5] (figure 1).

Si les épisodes suscités se sont déroulés dans les régions comprises entre les pays rhénans et la Wallonie, ils n'en eurent pas l'exclusivité. Des manifestations semblables eurent lieu en d'autres lieux et d'autres temps [4] : tarentisme en Italie et en Espagne aux XVI'-XVIII" siècles, zar en Abyssinie et en Égypte, ramanenjana ou tromba à Madagascar en 1863. Certains, comme ceux de la secte des Jumpers fondée en 1760 en Cornouaille ou de la secte des Chlustes (flagellants sauteurs de la Russie tsariste du XVIII' siècle), ont même cherché à en reproduire le phénomène qu'ils considéraient comme

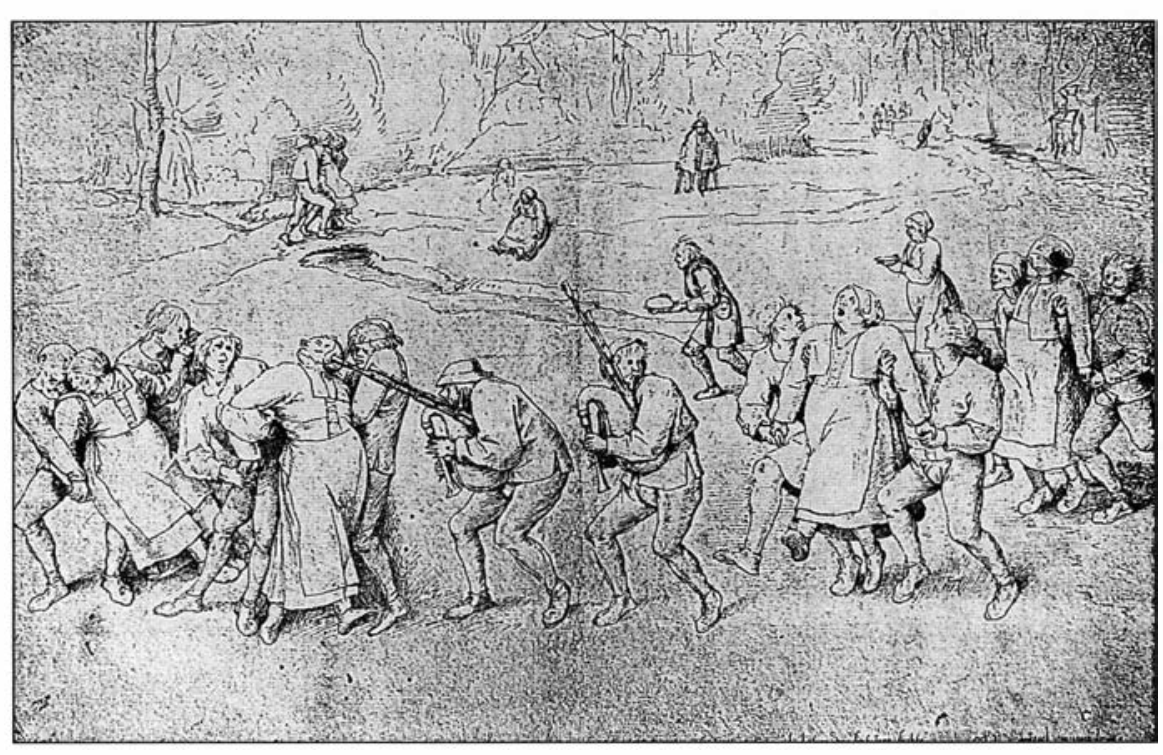

Figure 1. "Les pèlerins de la Saint Jean qui dansaient à Meulenbeeeck". Gravure d'après un dessin de Pieter Brueghel lavec l'autorisation du Bildarchiv der Österreichirschen). d'inspiration divine et non plus démoniaque.

Qu'est-ce qui a pu favoriser ces étranges épidémies? Il est aujourd'hui généralement admis que ces manifestations étaient sans base organique, mais de nature vraisemblablement hystérique. Plusieurs auteurs [3, 6, 7] ont souligné qu'un développement épidémique pouvait avoir lieu en cas de contexte de crise socio-culturelle majeure: difficiles conditions de vie, existences menacées, périodes de grands bouleversements sociaux avec perte ou conflit de valeurs. Ainsi l'histoire récente des "Français sauteurs du Maine" au Québec [20], présentant des myoclonies du sursaut suivies de danses, de cris et d'invocations durant de longues minutes. Un temps considérée comme une maladie de Gilles de la Tourette (tics) ou une chorée mineure, il semble qu'elle soit liée à des conduites de groupe dont on retrouve l'histoire parmi les communautés de bucherons au XIX" siècle. Dans de telles conditions, certains sujets finissaient par ressentir un besoin impérieux d'expression émotionnelle et motrice pour diminuer le stress accumulé. La danse du Moyen-Age n'avait donc probablement rien à voir avec la "chorée" définie par les médecins dans les siècles suivants. Elle la masquait sans doute, du fait de son caractère explosif (donc spectaculaire) et épidémique, alors que la chorée, peu fréquente et très dispersée, était hors des limites de détection d'un système médical très restreint et manquant de compétence.

\section{Description de la chorée par Sydenham}

Avec la Renaissance, la médecine d'observation s'amplifia rapidement et les chorées apparurent enfin, devenant objets médicaux. Philippus Aureolus Theophrastus Paracelsus Bombastus Von Hohenheim (14931541), mieux connu sous le nom de Paracelse, discuta le problème des chorées dans son livre sur les maladies invisibles, écrit vers 1531-1532 [4]. Le premier, il attribua clairement la chorea sancti Viti au pouvoir de la suggestion et des croyances,
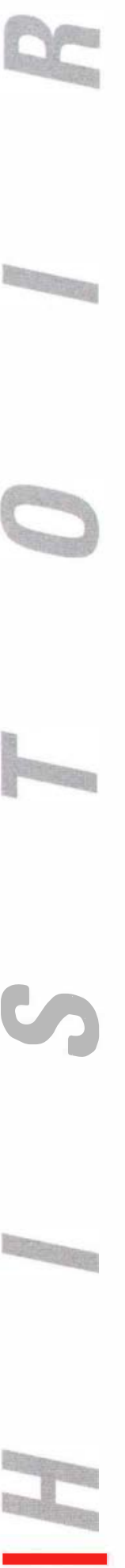

$m / s n^{\circ} 6-7$, vol. 13, juin-juillet 97 
«rien d'autre qu'une maladie imaginaire". Il en distingua, pour la première fois également, l'existence d'une forme organique, la chorea naturalis. C'est toutefois à Thomas Sydenham, «l'Hippocrate anglais", que l'on doit la première véritable description d'une chorée organique, dans son dernier ouvrage "Schedula Monitoria de Novae Febris Ingressa" (1686). Il y décrivit, en effet, une forme particulière de chorée "qui attaque garçons et filles de 10 ans et jusqu'à la fin de la puberté. En premier elle débute par des arrêts ou plutôt des mouvements irréguliers, instables d'une des jambes que le patient traîne, puis dans la main du même côté. Le patient ne peut pas la garder en place, quoiqu'il fasse elle sursautera ailleurs convulsivement.... Maintenant cette affection provient de quelques humeurs tombant sur les nerfs et une telle irritation cause le spasme » [8]. Cette description resta celle de la chorée de l'enfant, ou chorée mineure à laquelle son nom est toujours rattaché. Il faut noter que l'utilisation par Sydenham de l'ancienne terminologie chorea sancti viti fut malheureuse car elle pérennisa une certaine confusion terminologique.

En France, ces travaux furent popularisés par E.M. Bouteille, en 1810 seulement, dans son "Traité sur la chorée ". Il reconnut l'association entre la chorée de l'enfant et le rhumatisme articulaire, que Roger en 1866 compléta avec l'endocardite [9].

Une forme particulière: de l'adulte, chronique et héréditaire. La chorée de Huntington

Tout au long du XIX ${ }^{e}$ siècle, des médecins avaient attiré l'attention sur des formes cliniques de chorée apparaissant chez l'adulte et probablement héréditaires $[10,11]$ : Waters et Gordman (observations rapportées par Dunglison en 1842 et 1848) ; Lyon, en 1863 ; et Lund, médecin norvégien, qui publia de 1859 à 1868 trois articles dans une revue de son pays sur la chorée héréditaire. Il reconstitua les arbres généalogiques de deux familles avec

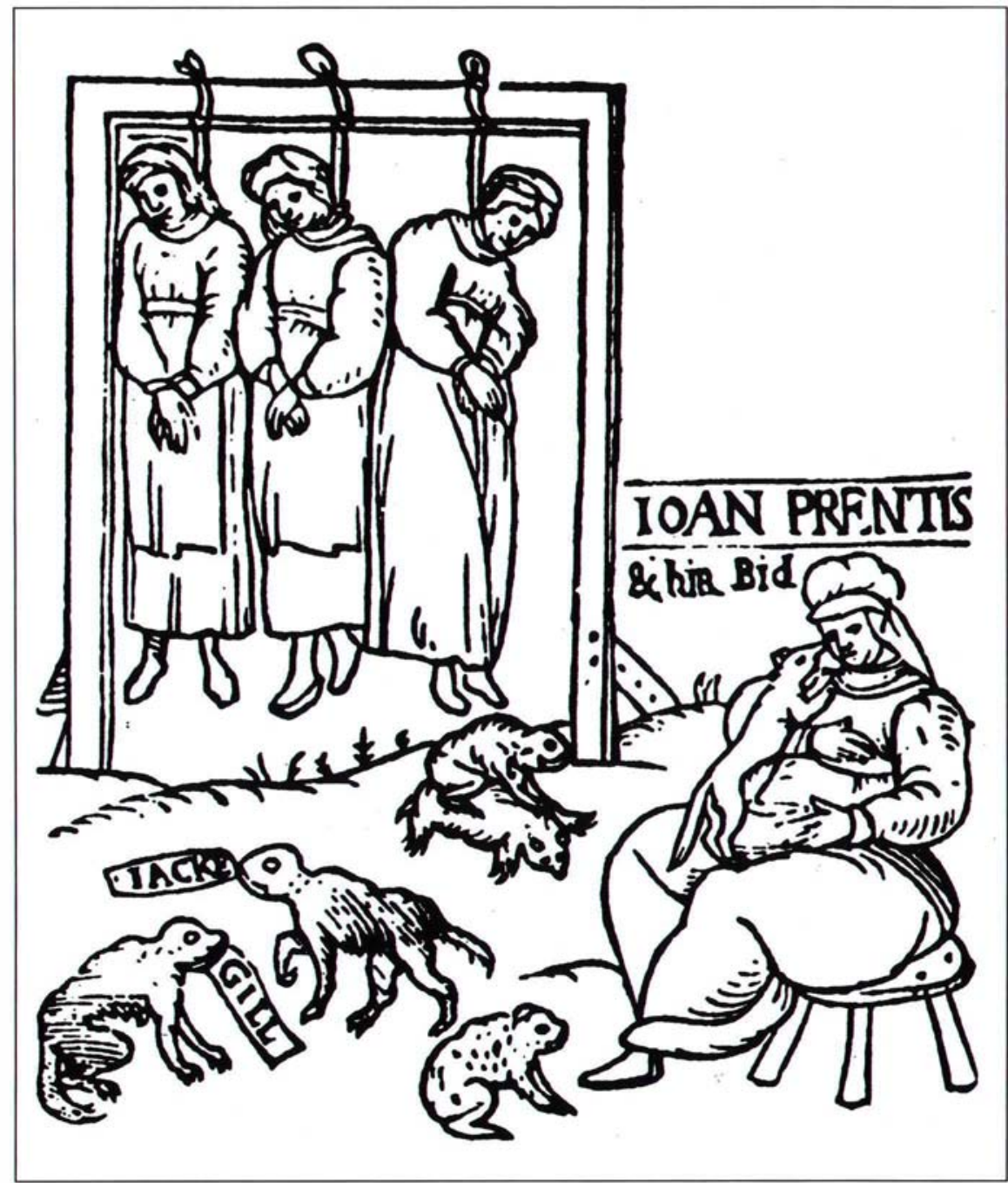

Figure 2. Pendaison de trois sorcières, dessin anonyme anglais, 1589.

Ses écrits restèrent toutefois largement méconnus, sans doute du fait de la barrière linguistique mais aussi à cause de l'erreur d'Hirsch, auteur d'un livre à succès en 1860, qui y rapportait l'affection décrite par Lund comme une paralysis agitans.

En fait, ce fut le 15 février 1872 que fut communiquée, par un jeune médecin de Long Island (dont ce fut d'ailleurs la seule contribution scientifique), la description qui fixa la véritable "date de naissance» de cette forme de l'adulte. Cette année-là, G.Huntington avait 22 ans, il exerçait depuis un an, lorsqu'il présenta sa thèse de médecine "On Chorea" devant l'Académie de Médecine Meigs and Masson de Middleport, Ohio, en présence d'un invité allemand de renom, le Pr Kussmaul. Ce fut au terme de son exposé qui portait essentiellement sur la chorée de Sydenham qu'il en décrivit une forme particulière, "héréditaire ", qu'il avait observé dès son enfance lorsqu'il accompagnait son père et dont l'intérêt et le savoir lui avait été transmis par deux générations successives de médecins installés à Hampton, Long Island, depuis 1797 [12]. Son mémoire parut aux États-Unis dans le Medical and Surgical Reporter [13] et fut remarquée par Kussmaul qui en fit un compte-rendu avec Nothnagel, cette même année dans le Virchov und Hirsch Jahresbericht, favorisant sa diffusion en Europe. La description 


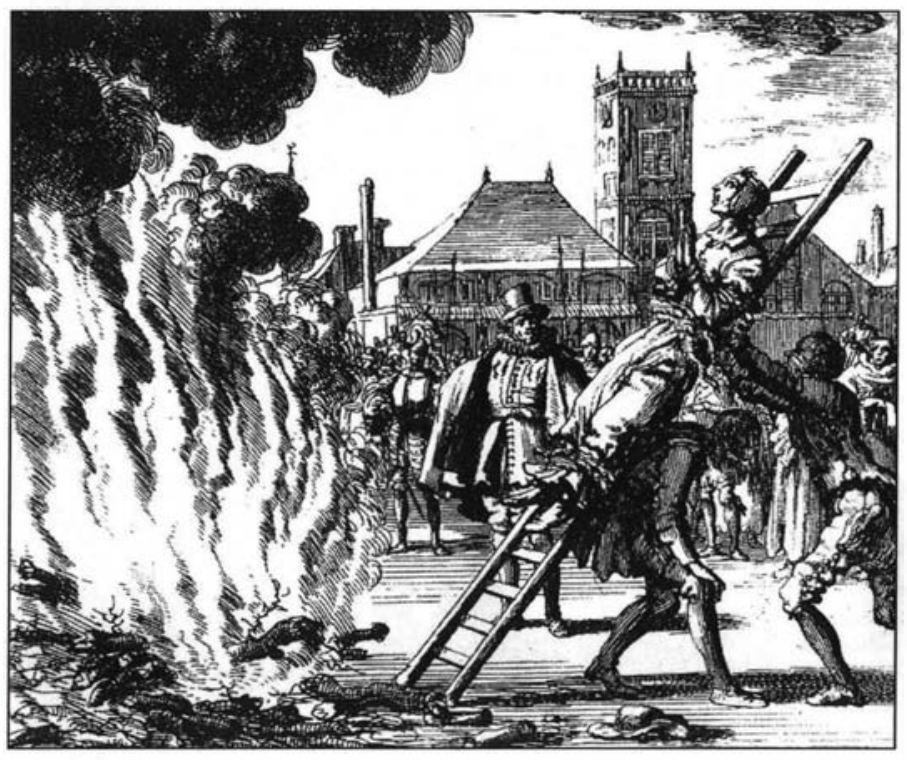

Figure 3. Supplice de Anne Hendricks, accusée de sorcellerie et brûlée à Amsterdam en 1571. Gravure d'époque. (Cl.RogerViollet.)

était si bonne qu'elle conduisit à cet éloge [14]: "Dans l'Histoire de la médecine il y a peu d'exemple où une maladie a été plus précisément, plus concisément décrite que celle par laquelle le Dr G. Huntington attire l'attention vers une chorée héréditaire qui prévaut dans l'extrême Est de Long Island...». G. Huntington débutait ainsi sa présentation:

"La maladie existe autant que je sache presque exclusivement à l'extrême Est de Long Island... La chorée héréditaire, comme je l'appellerai, est limitée à certaines familles, heureusement rares, et leur a été transmise tel un héritage des générations remontant à un très lointain passé. Elle présente tous les symptômes de la chorée commune mais à un niveau de gravité supérieur, survenant rarement avant l'âge adulte ou le milieu de la vie, s'aggravant graduellement mais sûrement pendant des années... Il y a trois particularités dans cette affection: sa nature héréditaire, sa tendance à la folie et au suicide, et sa gravité lorsqu'elle survient chez un adulte...»

Les travaux se multiplièrent et corroborèrent sa description qui se répandit rapidement. Les critères précis de diagnostic énoncés dès le départ permirent d'isoler une population homogène de patients atteints de la "chorée de Huntington". Le rôle de $\mathrm{m} / \mathrm{s} n^{\circ} 6-7$, vol. 13, juin-juillet 97

La description de G. Huntingto nante, provoqua très vite la mise en œuvre d'importantes recherches visant à reconstruire les arbres généalogiques des familles atteintes pour remonter vers l'origine de la maladie et, peut-être, trouver un effet fondateur. Rassemblant des cas éparses, appareillant des familles éloignées à un ancêtre commun ayant parfois vécu plusieurs siècles auparavant, les études généalogiques montrèrent que les pays neufs avaient été "contaminés " par des émigrants presque exclusivement ouest-européens. La chorée de Huntington fut ainsi, à rebours, extraite de l'ombre dans laquelle elle avait évolué jusque-là. Incidemment, ce travail aboutit à réhabiliter aux yeux de l'Histoire plusieurs malheureux malades que la foule avait, des siècles auparavant, pris pour des possédés. Ainsi, Vessie [15] identifia parmi les premiers américains atteints de chorée de Huntington, trois hommes, auxquels il donna les pseudonymes de Jeffers, Nicolas et Wilkie. Originaires de Bures, un petit village de l'Est de l'Angleterre, ils s'enfuirent vers la Nouvelle-Angleterre pour échapper à l'atmosphère d'oppression et aux persécutions pour sorcellerie, qui s'étaient intensifiées lors de l'accession au trône de Charles I (figures 2 et 3). Ils embarquèrent à Yarmouth en 1630 et arrivèrent trois mois plus tard à Salem. Malheureusement pour les nouveaux arrivants, dans les colonies d'Amérique aussi avait lieu une "chasse aux sorcières", principalement dans le Connecticut et le Massachusetts (en 1642 y fut votée une première loi antisorcellerie). Cette folie collective culmina en 1692 à Salem et aboutit à la condamnation à mort par pendaison (condamnation suivie d'effet!) de dix-neuf personnes. Au total, au moins 30 exécutions eurent lieu dans les territoires de NouvelleAngleterre au Xvi ${ }^{e}$ siècle, par pendaison ou sur le bûcher, et plusieurs éléments suggèrent que parmi ces infortunés figuraient des gens atteints de chorée de Huntington (sept femmes descendantes des trois hommes sus-cités furent alors considérées comme des sorcières) [16, 17]. Comme par coïncidence, un homme, né dans la même région d'Angleterre que Jeffers, Nicolas et Wilkie, suivit le même chemin trois ans plus tard. Il s'appelait Simon Huntington [18].

La maladie de Huntington entre actuellement dans une phase fort différente de son histoire. Avec le clona-
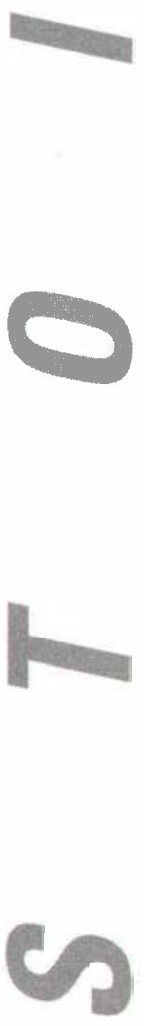

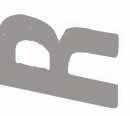 \\ $+$}


ge du gène $I T 15$ [19] et la mise en place d'un test génétique permettant l'identification des porteurs adultes mais aussi fotaux, la possibilité d'une quasi-éradication de cette maladie est offerte. Encore faut-il que les porteurs potentiels pré-symptomatiques aient un intérêt personnel à la connaissance de leur état. Or, la prise en charge médico-sociale reste très insuffisante. En effet, il n'existe toujours pas de thérapeutique validée, même symptomatique; quant à l'intégration sociale, si on doit admettre qu'elle a évolué puisque l'on ne brûle plus les malades, elle reste toutefois, comme c'est le cas dans de nombreuses affections neurologiques et psychiatriques, caractérisée par une détérioration intellectuelle et relationnelle, par la peur, et suivant le terme de notre époque, par l'exclusion.

L'histoire de la maladie de Huntington continuera sans doute encore, quelques temps, à révéler les relations complexes entre la médecine et la société dans laquelle elle se fait $\square$

Remerciements

Nous tenons à remercier tout particulièrement le Dr M. Peschanski pour ses précieux conseils.
RÉFÉRENCES

1. Martin A. History of dancing mania. $A m J$ Clin Med 1923; 30 : 265-71

2. Babonneix L. Quelques étapes dans l'histoire de la chorée. Le Monde médical 1925 $35: 852-67$.

3. Hecker JFC. The epidemics of the middle ages. London, 1862 : 87-107.

4. Schechter DC. St Vitus dance and rheumatic disease. NY St J Med 1975 : 1091-102.

5. Schullian DM. Notes and events: the dancing pilgrims at Muelebeek. I Hist Med 1977: $315-9$

6. De Felice P. Foules en délire, extases collectives: essai sur quelques formes inférieures de la mystique. Paris : Albin Michel, 1947:

7. Davidson A. Choreamania: an account of an epidemy observed in Madagascar in 1863. Edinb Med J 1967: 463-70.

8. Russell N, De Jong RN. Georges Hunting ton and his relationship to the earlier des criptions of chronic hereditary chorea. Ann Med Hist 1937; 9: 201-10.

9. Barbeau A. The understanding of involontary movements: an historical approach J Nerv Ment Dis 1958; 127 : 469-89.

10. Stevens DL. The history of Huntington's chorea. J Roy Coll Phys Lond 1972; 6: 271-82.

11. Orbeck AL. An early description of Huntington's chorea. Med Hist 1959; 3 : 165-8.

12. Huntington G. Recollections of Huntington's chorea as I saw it at East Hampton, Long Island, during my boyhood. NY Neurol Soc 1909: 255-7.

13. Huntington G. On chorea. Med Surg Rep 1872; 26: 317-21 (réédité dans Arch Neurol $1967 ; 17: 332-3)$.

14. Osler W. Historical note on hereditary chorea. Neurographs 1908; 1 : 113-6.
15. Vessie PR. On the transmission of Huntington's chorea for 300 years: the Bures family group. J Nerv Ment Dis 1932; 76: 533-73.

16. Hayden MR. Huntington's chorea. New York: Springer-Verlag, 1981 : 18.

17. Maltsberger JT. Even to the 12th generation: Huntington's chorea. J Hist Med 1961 ; 16: 1-17.

18. Critchley M. Huntington's chorea and East Anglia. J State Med 1934; 42: 575-87.

19. HDCRG. A novel gene containing a trinucleotide repeat that is expanded and unstable on Huntington's disease chromosomes. Cell 1993; 72: 971-83.

20. Saint-Hilaire MH, Saint Hilaire JM, Granger L. Jumping frenchmen of Maine. Neurology 1986; 36: 1269-71.

Thierry Grandmougin

Inserm U. 421, IM3, faculté de médecine, 8, rue du Général-Sarrail, 94010 Créteil Cedex, France.

\section{Catherine Bourdet}

Hôpital Albert-Chenevier, service de psychiatrie, 40, rue de Mesly, 94010 Créteil Cedex, France.

Jean-Marc Gurruchaga

Hôpital Henri-Mondor, service de neurologie, 51, avenue du Maréchal-de-Lattrede-Tassigny, 94010 Créteil Cedex, France.

\section{TIRÉS À PART}

T. Grandmougin.

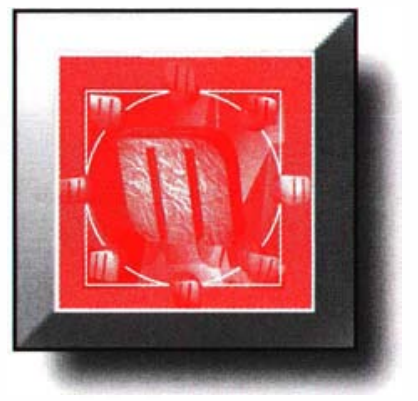

\section{Retrouvez les ReVUeS MASSON SUR INTERNET http://www.masson.fr e-mail : revues@masson.fr}

